\title{
LINC00311 is overexpressed in ankylosing spondylitis and predict treatment outcomes and recurrence
}

\author{
Hongfa Zhong and Mingliang Zhong*
}

\begin{abstract}
Background: LnCRNA LINC00311 participates in osteoporosis, which shows inverse pathological changes to ankylosing spondylitis (AS), indicating that LINC00311 is also involved in AS.

Methods: All the participants were enrolled in Ganzhou People's Hospital between January 2016 and January 2018 after this study was approved by Ganzhou People's Hospital Ethics Committee. Disease activity determination, follow-up and RT-qPCR were carried out during the research.

Results: In the present study we found that LINC00311 was upregulated in AS patients comparing to healthy controls, and upregulation of LINC00311 distinguished AS patients from healthy controls. LINC00311 expression levels were positively correlated with disease activity. Comparing to pre-treatment levels, LINC00311 expression level decreased significantly after treatment. During 2-year follow-up, patients with high levels of LINC00311 showed a significantly higher rate of rehospitalization.
\end{abstract}

Conclusions: Therefore, LINC00311 is overexpressed in AS and predict treatment outcomes and recurrence.

Keywords: Ankylosing spondylitis, IncRNA LINC00311, Re-hospitalization

\section{Introduction}

Ankylosing spondylitis (AS) is a type of chronic inflammatory rheumatic disease that mainly affects skeletal system [1]. AS not only causes chronic back pain but may also result in functional and structural impairments [1]. As a common type of clinical disorder AS affects about 1 out of 110 people in western countries and about 1 out of 200 people in China [2, 3]. In spite of the efforts made on the treatment of AS, prognosis of AS is poor and most AS patients will experience recurrence within a short-term after discharge $[4,5]$. Therefore, accurate prognosis is needed to predict the development of AS.

Genetic factors, such as the altered gene expression, contribute to the development of progression of AS [6, 7]. Besides protein players, non-coding RNAs, such as long non-coding RNAs (> $200 \mathrm{nt}$, lncRNAs) have also been characterized as critical regulators in human

\footnotetext{
* Correspondence: swmnne0069617@126.com

Department of orthopaedics, Ganzhou People's Hospital, No.16 Meiguang

Avenue, Ganzhou City, Jiang Xi Province 341000, People's Republic of China
}

disease development and progression due to their roles in regulating gene expression at multiple levels $[8,9]$. Analysis of the expression pattern and functions of IncRNAs may contribute to disease treatment and prediction $[8,9]$, while function of most lncRNAs remains unclear. A recent study reported that lncRNA LINC00311 participates in osteoporosis by regulating the differentiation and proliferation of osteoclasts [10]. It is known that osteoporosis and AS have inverse pathological changes [11]. Our study therefore was carried out to investigate the involvement of LINC00311 in AS.

\section{Methods}

Research patients

Patient group in this study included 80 AS patients (47 males and 33 females, 24 to 46 years, $30.3 \pm 4.8$ years). Inclusion criteria: 1) newly diagnosed AS patients; 2) no therapies initiated before admission. Exclusion criteria: 1) other clinical disorders were observed; 2) with previous history of malignancies; 3 ) with family history of malignancies. Control group included 80 healthy volunteers 
(48 males and 32 females, 23 to 47 years, $32.1 \pm 4.9$ years) and 22 low back pain (LBP) patients (12 males and 10 females, 24 to 49 years, $31.9 \pm 5.1$ years). Controls and LBP patients were included to match the distributions of age and gender of AS patient group. Controls with previous or family history of malignancies were excluded. All the participants were enrolled in Ganzhou People's Hospital between January 2016 and January 2018 after this study was approved by Ganzhou People's Hospital Ethics Committee. All patients and controls signed written informed consent.

\section{Disease activity determination}

Both Ankylosing Spondylitis Disease Activity Index (BASDAI) and The Ankylosing Spondylitis Disease Activity Score (ASDAS) 1-4 were used to determine the disease activity of all 80 AS patients. Levels of inflammatory markers $C$ reactive protein (CRP) and Estrogen Receptor (ESR) in plasma were measured with ELISA using Human C Reactive Protein ELISA Kit (CRP) (ab99995, Abcam) and human Estrogen Receptor Alpha ELISA Kit (ab128499, Abcam), respectively.

\section{Plasma and treatment}

Before any therapies were initiated, blood ( $5 \mathrm{ml}$ ) was extracted from each participant under fasting conditions. Blood was centrifuged at room temperature for $2 \mathrm{~h}$ at $1200 \mathrm{~g}$ to prepare plasma samples.

All patients (AS and low back pain) were treated with nonsteroidal anti-inflammatory drugs, such as indomethacin and naproxen (doses varies across patients based on their disease conditions). The relieved pain, inflammation and stiffness indicated the relieved disease conditions (reflected by BASDAI and ASDAS scores).

At 2 months after the initiation of treatment (significantly improved BASDAI and ASDAS were observed at this time point), blood $(5 \mathrm{ml})$ was extracted from each patient under fasting conditions to prepare plasma samples using aforementioned methods.

\section{Follow-up}

The 80 AS patients were monitored for 2 years after admission. Follow-up was performed through phone call or outpatient visit. The cases of re-hospitalization were recorded and analyzed.

\section{RT-qPCR}

Plasma samples were mixed with Ribozol reagent (Thermo Fisher Scientific) to extract total RNAs. Total RNAs were used as template to perform reverse transcriptions using First Strand cDNA Synthesis Kit for RTPCR (AMV, Sigma-Aldrich, USA). To detect the expression of LINC00311, qPCR reaction mixtures were prepared using Invitrogen SuperScript ${ }^{\oplus}$ III Platinum ${ }^{\odot}$ SYBR $^{\oplus}$
Green One-Step qRT-PCR Kit (Thermo Fisher Scientific) with $18 \mathrm{~S}$ rRNA as endogenous control. All PCR reactions were performed 3 times and data were normalized using $2^{-\Delta \Delta \mathrm{CT}}$ method.

\section{Statistical analysis}

Mean values presented in this paper were from 3 biological replicates of each experiment. Differences among 3 groups were analyzed by performing ANOVA (oneway) and Tukey test. Differences between pre- and posttreatment levels of LINC00311 in AS patients were analyzed by performing paired test. Diagnostic values of LINC00311 for AS were analyzed by performing ROC curve analyses. In ROC curve analysis, AS patients were true positive controls and healthy controls were true negative controls. Comparisons of re-hospitalization rates were analyzed by performing Chi-squared test. Correlations were analyzed by performing linear regression analysis. Statistically significant level was $p<0.05$.

\section{Results}

\section{Plasma LINC00311 was upregulated in AS}

LINC00311 in plasma of AS patients $(n=80)$, LBP patients $(n=22)$ and controls $(n=80)$ was detected by performing RT-qPCR experiments. Plasma levels of LINC00311 were compared by ANOVA (one-way) and Tukey test. It was observed that plasma levels of LINC00311 were significantly higher in AS patients comparing to controls and LBP patients (Fig. 1, $p=$ 0.012 and 0.011 , respectively). This finding suggest that altered expression of LINC00311 is likely involved in the pathogenesis of AS.

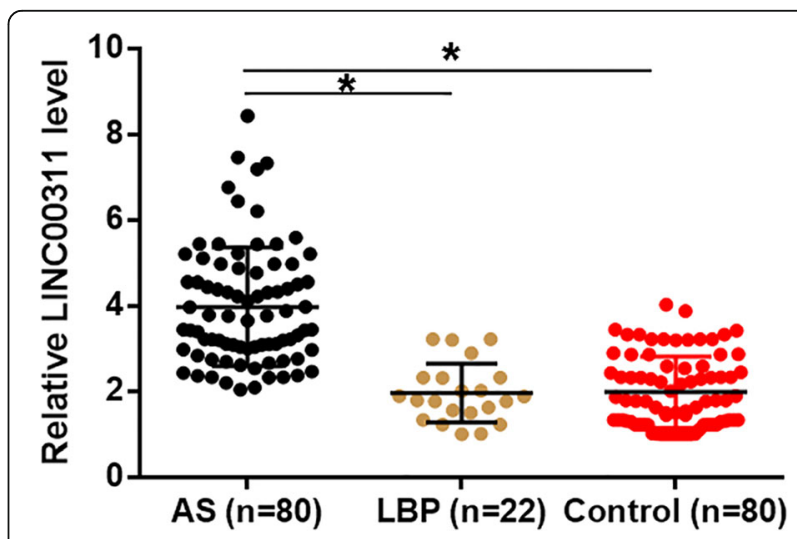

Fig. 1 Plasma LINC00311 was upregulated in AS. LINC00311 in plasma of was detected by RT-qPCR and expression data were compared by performing ANOVA (one-way) and Tukey test. It was observed that plasma levels of LINC00311 were significantly higher in AS patients than in controls and LBP patients ( $\left.{ }^{*}, p<0.05\right)$ 


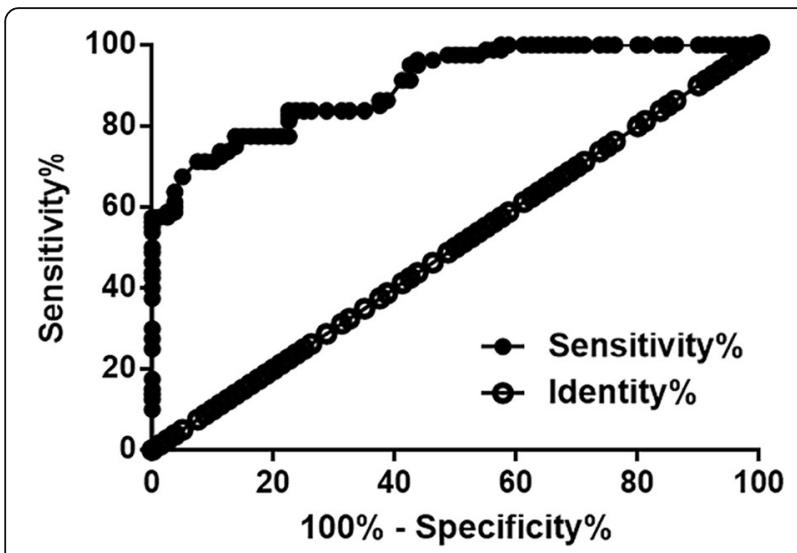

Fig. 2 Upregulated plasma levels of LINC00311 showed diagnostic values for AS. ROC curve analysis showed that upregulated plasma levels of LINC00311 distinguished AS patient from healthy controls

\section{Upregulated plasma levels of LINC00311 showed} diagnostic values for AS

Diagnostic values of LINC00311 for AS were analyzed by performing ROC curve analyses. In ROC curve analysis, AS patients were true positive controls and healthy controls were true negative controls. As shown in Fig. 2, area under the curve was 0.9041 , with standard error of 0.02268 and $95 \%$ confidence interval of $0.8569-0.9459$ (Fig. 2, $p<0.0001$ ). Therefore, plasma LINC00311 may be a potential diagnostic marker for AS.

\section{LINC00311 expression levels were positively correlated with disease activity}

BASDAI and ASDAS 1-4 were widely used to determine the activities of AS. In addition, levels of CRP and ESR are also used to reflect the inflammation in AS patients. In this study all patients were subjected to BASDAI and ASDAS 1-4 scoring and plasma levels of CRP and ESR in AS patients were measured by performing ELISA. Linear regression was performed to analyze the correlations between those markers and plasma levels of LINC00311. As shown in Table 1, BASDAI and ASDAS
1-4 were significantly and positively correlated with CRP and ESR ( $\mathrm{R}$ square $>0.65$, all $p$ values $<0.0001$ ). In addition, BASDAI, ASDAS 1-4, CRP and ESR were also significantly and positively correlated with plasma levels of LINC00311 ( $\mathrm{R}$ square $>0.65$, all $p$ values $<0.0001$ ).

\section{LINC00311 levels in plasma decreased after treatment}

Plasma levels of LINC00311 in AS patients were measured both before and 2 months after the initiation of treatment. Plasma levels of LINC00311 were compared by performing paired $t$ test between 2 time points. Comparing to pre-treatment levels, plasma levels of LINC00311 were significantly reduced at 2 months after the initiation of treatment (Fig. 3, $p=0.017$ ).

\section{Plasm levels of LINC00311 before treatment predicted re-} hospitalization

The 80 patients were divided into high $(n=37)$ and low $(n=43)$ LINC00311 level groups according to Youden's index. During the 2-year follow-up, 39 patients were rehospitalized, including 28 cases in high LINC00311 level group $(75.7 \%)$ and 11 cases in low LINC00311 level group (25.6\%). Comparisons of re-hospitalization rates were analyzed by performing Chi-squared test. It was observed that patients with high levels of LINC00311 showed a significantly higher rate of re-hospitalization (Fig. 4, chi-square statistic $=19.9751, p<0.011$ ).

\section{Discussion}

We analyzed the expression pattern of LINC00311 in AS and also explored the clinical significance of altered plasma levels of LINC00311 for AS. We found LINC00311 can be used to assist the diagnosis and prognosis of AS.

Previous studies have identified quite a few lncRNAs related to AS. Li et al. reported that lncRNA AK001085 was downregulated in AS, and the downregulation of AK001085 effectively separated AS patients from healthy controls [12]. In another study, Lan et al. found that the reduced expression of lncRNA TUG1 was closely correlated with the course of treatment and disease activity of

Table 1 LINC00311 expression levels were positively correlated with disease activity (Linear regression of BASDAI, ASDAS 1-4, CRP and ESR were also significantly and positively correlated with plasma levels of LINC00311)

\begin{tabular}{|c|c|c|c|c|c|c|c|}
\hline \multirow[t]{2}{*}{ Markers } & \multirow[t]{2}{*}{ Cases } & \multicolumn{2}{|l|}{ ESR } & \multicolumn{2}{|l|}{ CRP } & \multicolumn{2}{|c|}{ LINC00311 } \\
\hline & & R square & $p$ value & R square & $p$ value & R square & $p$ value \\
\hline BASDAI & 80 & 0.89 & $<0.0001$ & 0.91 & $<0.0001$ & 0.86 & $<0.0001$ \\
\hline ASDAS 1 & 80 & 0.89 & $<0.0001$ & 0.88 & $<0.0001$ & 0.87 & $<0.0001$ \\
\hline ASDAS 2 & 80 & 0.90 & $<0.0001$ & 0.88 & $<0.0001$ & 0.84 & $<0.0001$ \\
\hline ASDAS 3 & 80 & 0.93 & $<0.0001$ & 0.92 & $<0.0001$ & 0.84 & $<0.0001$ \\
\hline ASDAS 4 & 80 & 0.89 & $<0.0001$ & 0.93 & $<0.0001$ & 0.85 & $<0.0001$ \\
\hline ESR & 80 & 1.00 & $<0.0001$ & 0.94 & $<0.0001$ & 0.84 & $<0.0001$ \\
\hline CRP & 80 & 0.94 & $<0.0001$ & 1.00 & $<0.0001$ & 0.89 & $<0.0001$ \\
\hline
\end{tabular}




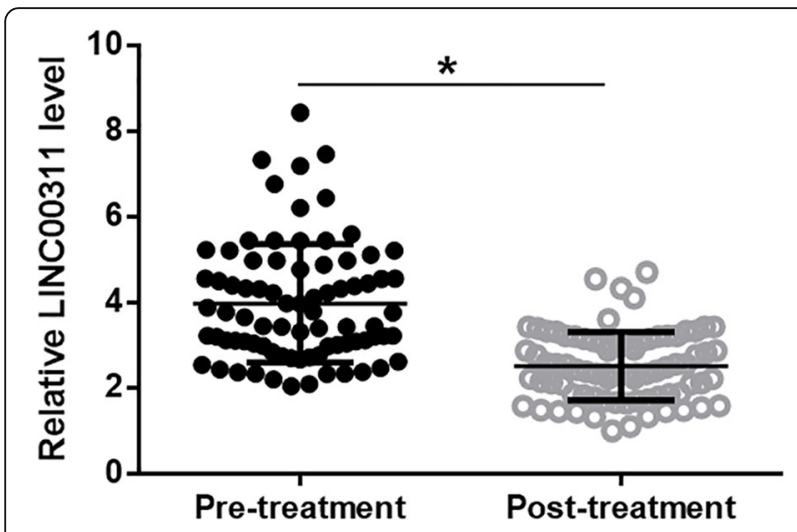

Fig. 3 LINC00311 levels in plasma decreased after treatment. Plasma levels of LINC00311 were compared by performing paired t test between 2 time points. It was observed that, comparing to pretreatment levels, plasma levels of LINC00311 were significantly reduced at 2 months after the initiation of treatment $(*, p<0.05)$

AS patients [13]. In a recent study, IncRNA MEG3 was found to be downregulated in AS and is associated with hospitalization time, disease activity and disease duration [14]. A recent study reported that IncRNA LINC00311 participates in osteoporosis [10], which has inverse pathological changes to AS [11], indicating the potential involvement of LINC00311 in AS. In the present study we proved that LINC00311 was upregulated in AS and may serve as a potential diagnostic biomarker for AS. However, the expression pattern of LINC00311 in other human diseases is unknown, so the sensitivity and specificity are still questionable.

BASDAI and ASDAS were widely used to asses AS activity [15]. In addition, levels of CRP and ESR are also

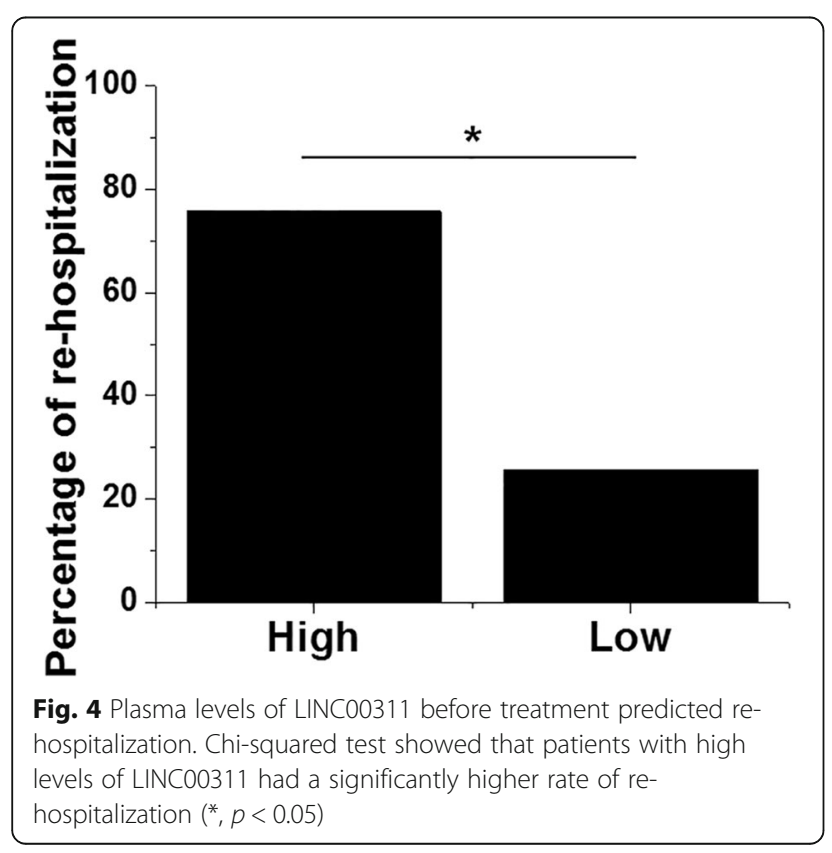

used to reflect the inflammation in AS patients [16, 17]. In the present study we showed that BASDAI and ASDAS 1-4 were significant and positively correlated with plasma levels of CRP and ESR in AS patients, indicating that BASDAI and ASDAS can be used to reflect the in vivo inflammation conditions of AS patients. In addition, plasma levels of LINC00311 were also significantly and positively correlated with BASDAI and ASDAS scores as well as plasma levels of CRP and ESR. Therefore, plasma LINC00311 may serve as a new biomarker of AS activity.

Besides diagnostic potentials and the correlation with disease acidity, our study also focused on rehospitalization, which is extremely common among AS patients [7]. We found that high plasma levels of LINC00311 were accompanied by high rehospitalization rate. Therefore, measurement of plasma levels of LINC00311 before treatment may be used to predict its recurrence, and individualized treatment strategies may be developed based on plasma levels of LINC00311 to reduce the recurrence rate.

\section{Conclusion}

In conclusion, LINC00311 was upregulated in AS, and upregulated LINC00311 has clinical potentials for the diagnosis and prognosis of AS.

\section{Abbreviations}

AS: Ankylosing spondylitis; ASDAS: Ankylosing Spondylitis Disease Activity Score; BASDAl: Bath Ankylosing Spondylitis Disease Activity Index; CRP: C reactive protein; ESR: Estrogen Receptor; IncRNAs: Long non-coding RNAs

\section{Acknowledgements}

Not applicable.

Authors' contributions

ZHF: analyzed and interpreted the patient data, experiments work and manuscript writing. ZML: literature research, research design and manuscript editing. All authors read and approved the final manuscript.

Funding

No funding was obtained for this study.

Availability of data and materials

The datasets used and/or analyzed during the current study are available from the corresponding author on reasonable request.

Ethics approval and consent to participate

Ethical approval was obtained from the Ethics Committee of Ganzhou People's Hospital. All procedures performed in studies involving human participants were in accordance with the ethical standards of the institutional and national research committee. Written informed consent was obtained from all individual participants included in this study.

\section{Consent for publication}

Not applicable.

\section{Competing interests}

The authors declare that they have no competing interests. 
Received: 15 March 2019 Accepted: 20 May 2019

Published online: 07 June 2019

\section{References}

1. Braun J, Sieper J. Ankylosing spondylitis. Lancet. 2007;369(9570):1379-90.

2. Braun J, Bollow M, Remlinger $G$, Eggens $U$, Rudwaleit $M$, Distler $A$, et al. Prevalence of spondylarthropathies in HLA-B27 positive and negative blood donors. Arthritis Rheum. 1998;41(1):58-67.

3. Zeng QY, Chen R, Darmawan J, Xiao ZY, Chen SB, Wigley R, et al. Rheumatic diseases in China. Arthritis Res Ther. 2008;10(1):R17

4. Braun J, Pincus T. Mortality, course of disease and prognosis of patients with ankylosing spondylitis. Clin Exp Rheumatol. 2002;20(6 Suppl 28):S16-22.

5. Lee JH, Choi M, Rim THT, Lee SC, Lee CS. Clinical characteristics and prognostic factors in ankylosing spondylitis associated uveitis. Ocul Immunol Inflamm. 2017;27(1):64-9.

6. Hanson A, Brown MA. Genetics and the causes of ankylosing spondylitis. Rheum Dis Clin N Am. 2017:43(3):401-14.

7. Brown MA, Kenna T, Wordsworth BP. Genetics of ankylosing spondylitis-insights into pathogenesis. Nat Rev Rheumatol. 2016;12(2):81-91.

8. Lalevee S, Feil R. Long noncoding RNAs in human disease: emerging mechanisms and therapeutic strategies. Epigenomics. 2015;7(6):877-9.

9. Kwok ZH, Tay Y. Long noncoding RNAs: lincs between human health and disease. Biochem Soc Trans. 2017:45(3):805-12.

10. Wang Y, Luo TB, Liu L, Cui ZQ. LnCRNA LINC00311 promotes the proliferation and differentiation of osteoclasts in osteoporotic rats through the notch signaling pathway by targeting DLL3. Cell Physiol Biochem. 2018; 47(6):2291-306.

11. Hinze AM, Louie GH. Osteoporosis Management in Ankylosing Spondylitis. Curr Treatm Opt Rheumatol. 2016;2(4):271-82.

12. Li X, Chai W, Zhang G, Ni M, Chen J, Dong J, et al. Down-regulation of IncRNA-AK001085 and its influences on the diagnosis of ankylosing spondylitis. Med Sci Monit. 2017;23:11-6.

13. Lan X, Ma H, Zhang Z, Ye D, Min J, Cai F, et al. Downregulation of IncRNA TUG1 is involved in ankylosing spondylitis and is related to disease activity and course of treatment. Biosci Trends. 2018;12(4):389-94.

14. Liu W, Huang L, Zhang C, Liu Z. InCRNA MEG3 is downregulated in ankylosing spondylitis and associated with disease activity, hospitalization time and disease duration. Exp Ther Med. 2019;17(1):291-7.

15. Machado P, Landewe R, Lie E, Kvien TK, Braun J, Baker D, et al. Ankylosing spondylitis disease activity score (ASDAS): defining cut-off values for disease activity states and improvement scores. Ann Rheum Dis. 2011;70(1):47-53.

16. Zochling J. Measures of symptoms and disease status in ankylosing spondylitis: ankylosing spondylitis disease activity score (ASDAS), ankylosing spondylitis quality of life scale (ASQoL), Bath ankylosing spondylitis disease activity index (BASDAI), Bath ankylosing spondylitis functional index (BASFI), Bath ankylosing spondylitis global score (BAS-G), Bath ankylosing spondylitis metrology index (BASMI), Dougados functional index (DFI), and health assessment questionnaire for the Spondylarthropathies (HAQ-S). Arthritis Care Res (Hoboken). 2011;63(Suppl 11):S47-58

17. Spoorenberg A, van der Heijde D, de Klerk E, Dougados M, de Vlam K Mielants $\mathrm{H}$, et al. Relative value of erythrocyte sedimentation rate and $\mathrm{C}$ reactive protein in assessment of disease activity in ankylosing spondylitis. J Rheumatol. 1999;26(4):980-4

\section{Publisher's Note}

Springer Nature remains neutral with regard to jurisdictional claims in published maps and institutional affiliations.

Ready to submit your research? Choose BMC and benefit from:

- fast, convenient online submission

- thorough peer review by experienced researchers in your field

- rapid publication on acceptance

- support for research data, including large and complex data types

- gold Open Access which fosters wider collaboration and increased citations

- maximum visibility for your research: over $100 \mathrm{M}$ website views per year

At $\mathrm{BMC}$, research is always in progress.

Learn more biomedcentral.com/submissions 